\title{
2006-108: DEVELOPING AN ENERGY MECHATRONICS LABORATORY
}

Henry Foust, Nicholls State University 


\title{
Developing an Energy Mechatronics Laboratory
}

\author{
March 8, 2006
}

\begin{abstract}
Oil forms a major component of U.S. energy policy and economy. It is imperative today to determine viable alternatives to oil (expected to be depleted within the next fifty years), because the infrastructure for a viable alternative will take decades to develop. The purpose of this research is the design of a mechatronics laboratory applied to the broad area of system analysis and control for Ocean Thermal Energy Conversion (OTEC), which, among other things, is the transformation of the huge sources of thermal energy trapped in oceans into electrical energy. The energy mechatronics laboratory will ask broad questions about the performance of particular OTEC plants, the required infrastructure for this technology, the economic environment needed to make this technology viable, and the use of OTEC for hydrogen generation.

OTEC is an area that was an emerging alternative energy source in the 1970's, but research petered out as a result of the price for oil dropping by 1980. But now, with the price of oil surging, a renewal of interest in OTEC has begun $[2,3,4]$. Much of the research emphasis is occurring outside of the U.S.

But what is mechatronics and its role in this research? Mechatronics is the application of electrical and computer principles to mechanical systems [1]. One example is the application of sensor, actuator, and micro-controllers to automotive engines. Mechatronics can be used in alternative energy conversions in respect to sensor selection, actuators, control methods, and the implementation of optimization schemes where a system approach develops the dynamic models and constraints on the optimization.

Another component of the research is that the mechatronics laboratory works in conjunction with a material science laboratory for a summer undergraduate research experience. The governing idea is to take the students through the process of writing a publishable research article. Steps in this process include background on understanding our energy needs, projected future needs, oil reserve estimates, and alternative energies; understanding the economics of energy; identifying viable alternatives; developing an argument for that viability; testing this argument through lab work; presenting the argument, method, and results; and preparation of a research paper.

The format of this research experience is a paid 12-week, 40-hour per a week internship. The breakdown of this internship is the following: 4 weeks of lectures,
\end{abstract}


2 weeks of developing a method to argue this thesis, and 6 weeks to test the argument and present results. The first four weeks will be an intensive lecture period; the second period will be an intensive seminar period; while the third period, will be laboratory and computer work. Lectures will be given on energy consumption and sources to fulfill these needs; generation of energy; viable alternative energy sources; distribution of energy; economics of energy; mechatronics; process of selecting a problem statement; methods of testing a thesis; and writing and presentation. Seminar and laboratory exercises will flow from the needs identified in the first four weeks.

\section{Introduction}

The Nicholls State University Department of Applied Sciences curriculum includes classes on mechatronics and a mechatronics laboratory. Observations from this class and laboratory form the basis for a proposed undergraduate research experience. This research experience would be a 12-week summer program for undergraduates to conduct research in using mechatronics principles toward improving alternative energy generation, conversion, or storage. The department is in the process of applying for a grant to fund the energy mechatronics laboratory.

\subsection{Mechatronics Class and Laboratory}

The department's class and laboratory on mechatronics teaches the application of electrical and computer principles to mechanical systems. This class began with lectures on DC circuits, linear system theory, and AC circuits. It then went into lectures on sensors, actuators, and data acquisition. One of the last areas covered was the introduction of programmable logic controllers through the use of LogixPro. LogixPro is a software program that simulates Allen Bradley's RSLogix 500 and includes a programmable process simulator.

The final stage of the laboratory and extra credit for the class was a team project. A list of the projects are

- Bucket Project

- Hydroelectric Design

- Windmill

- Digital Tachometer

The bucket project was a project where water was entering and leaving a bucket and through sensors and actuators a constant water level was maintained. This project in- 
cluded two component: a practical component and a theory component. The theory component group designed the systems to include the use of state feedback and PID control. The practical component group built it. The windmill project required the students to built a windmill that drove an LED. The hydroelectric design was where the students designed a $150 \mathrm{~kW}$ plant. The design included the penstock, turbine, and an extensive economic analysis. The last project was the development of a digital tachometer, which measures the $\operatorname{rpm}(\mathrm{s})$ of a 4 -stroke engine.

Observations from the mechatronics class and laboratory include:

- Students learn more in a hands-on environment

- Lectures, although important to establish fundamental understandings, can deaden the imagination

- Students learn the most when the environment is creative

These observations were utilized to develop the proposed summer undergraduate research experience.

\subsection{Energy Mechatronics Laboratory}

The format of this research experience is a paid 12-week, 40-hour per a week internship. The breakdown of this internship is the following: 4 weeks of lectures, 2 weeks of developing a method to argue this thesis, and 6 weeks to test the argument and present results. The first four weeks will be an intensive lecture period; the second period will be an intensive seminar period; while the third period, will be laboratory and computer work. Lectures will be given on energy consumption and sources to fulfill these needs; generation of energy; viable alternative energy sources; distribution of energy; economics of energy; mechatronics; process of selecting a problem statement; methods of testing a thesis; and writing and presentation. Seminar and laboratory exercises will flow from the needs identified in the first four weeks.

\section{Methodology}

The methodology for this summer research experience includes four components: curriculum, assessment, experimental medium, and likely outcomes. Each of these areas is discussed in the sections below. 


\subsection{Curriculum}

The curriculum for this 12 week laboratory includes four distinct parts, which are shown in Table 1. The first part is 4 weeks of lectures; the second part is 2 weeks of developing a thesis and a method to argue that thesis; the third part is verifying the thesis; and the last part is to present the findings of the study. Each part is discussed below.

The purpose of the research experience is to have students develop a thesis and successfully argue that thesis through computational or experimental results. Specific goals will be centered around four reports, which form the basis to a research paper.

The four reports are a literature review, thesis and methodology, results, and discussion/conclusions. These four reports together will form a research paper.

\subsubsection{Part I - Lectures}

The lecture part will be 4 days per the 5 day work week, which excludes Friday. Mornings will be 3 hours of lecture and afternoons will be the time provided to work homeworks.

Fridays, through out the research experience, will be utilized for meetings. First, the groups will meet, which will have been formed on the first day, and discuss the weeks events. Then each group will present a given topic for the other groups. The last meetings will be between individual students and mentors.

Four topics will be discussed (see Table 1) fluid mechanics, thermodynamics, economics, and energy systems. It is a pre-requisite that students have taken fluid mechanics and thermodynamics, because the fluid mechanics and thermodynamics taught will be of an advanced nature.

\subsubsection{Part II - Seminars}

The seminar part will be 4 days per the 5 day work week with seminars occupying 3 hours of the morning. Afternoons will be used for homework completion and field trips.

\subsubsection{Part III - Experimentation}

The students have decided upon a problem statement, methodology, and are free to spend their weeks as they see fit with guidance from mentors as requested. Guidance will come in the form of weekly meetings to present that weeks findings, a weekly meeting with their mentor, and additional guidance as determined. 
Table 1: Course Outline

\begin{tabular}{|l|l|l|}
\hline Week & Mode & Activities \\
\hline 1 & Lecture & Thermodynamics \\
2 & Lecture & Fluid Mechanics \\
3 & Lecture & Economics \\
4 & Lecture & Energy Systems \\
5 & Seminar & Problem Statement \\
6 & Seminar & Methods \\
7 & Experiment & As needed \\
8 & Experiment & As needed \\
9 & Experiment & As needed \\
10 & Experiment & As needed \\
11 & Experiment & As needed \\
12 & Presentation & As needed \\
\hline
\end{tabular}

\subsubsection{Part IV - Presentation}

This week will be utilized to prepare a final report and presentation. The final report is a six page conference paper.

\section{$2.2 \quad$ Assessment}

The first assessment tool is to determine the students' ability prior to the first day of the research experience through a survey, interview, and essay to insure each group has a good balance of communication and analytical skills.

The assessment of how well the program is working is how well the student is doing. To gauge this success, several tools will be utilized (see Table 2). These tools include likely meetings where each team gives a presentation, team grades, homework assignments, a graded diary, and project grade. Each of these areas will be discussed.

Homeworks are utilized to ensure the students have the basics. A research journal is utilized to ensure the student maintains disciplined records of the methods developed and experiments conducted. The project grade actually represents four grades (each worth 100 points) that are

- Problem Statement

- Methodology

- Results 
Table 2: Grading System

\begin{tabular}{|l|l|}
\hline Assignment & Grading Value \\
\hline Homework & 350 \\
Team Grade & 150 \\
Research Journal & 100 \\
Project & 400 \\
\hline
\end{tabular}

- Presentation

\subsection{Experimental Medium}

Students have a choice between doing experimental work or computational work. The experimental work will center around using LabView, which is an instrumentation package. The computational work will center around using MathCad or Matlab, which are computational packages.

The application of these media to the research will be further discussed in the energy mechatronics laboratory is discussed, which occurs below.

\subsection{Likely Outcomes}

The likely outcomes include that the students have an understanding of how complicated energy issues are, an idea on how to develop a thesis and argue that thesis, and a draft to a conference paper. It is unlikely that within 12 weeks the students will have conducted enough research and gotten the results for a publishable paper, but they will have seen the research process.

If research does result in a publishable conference paper, the student and mentor will work throughout the regular year to turn the draft into a publishable conference paper and present the paper at an identified conference.

\section{Energy Mechatronics Laboratory}

The research to be conducted will center around three issues: material science issues associated with intake pipes, increasing the thermal efficiency, and environmental issues associated with heat pollution. Each of these topics will be discussed in the paragraphs below. 
What are the best materials to use for the intake pipes that deliver water to the OTEC turbine and how to best minimize the effects of a marine environment, which is highly corrosive, is the topic of this area of research. What is meant by 'best' is to reduce the heat transfer to the environment and minimize corrosiveness. This research area will include material property testing and possible simulations, which would entail using both LabView and MathCad (Matlab).

A more theoretical focus is to ask what is the best way to run an OTEC plant to maximize the energy generation, minimize environmental impacts, and reduce capital costs. This research would involve more mathematical sophistication and could later lead to collaborations with mathematics departments here at Nicholls State University and across the state. This would entail using MathCad (Matlab).

The third area of research is to explore the heat pollution generated by OTEC and it's impact on marine biota. This focus forms a bridge between the Biological Science and Applied Science departments at Nicholls State University. This would either entail experimental or computational work, which entails the use of both LabView and MathCad (Matlab).

\section{Discussion}

The student will complete the summer undergraduate research experience and have money, draft of a publishable paper, and college credit to show for their time. They will also have the invaluable experience of having gone through a complete research experience and having made a significant contribution to their chosen field of research.

As can be seen from the testimony of the mechatronics class and laboratory, an openended problem can be both frustrating and rewarding. It is up to the mentor to determine how much to expect from each group and how to keep them on track.

A similar experience is being proposed as a NSF grant for an energy mechatronics laboratory. This research will implement the methods developed for the mechatronics class and laboratory within an energy mechatronics setting where a current research area is tackled.

\section{Conclusions}

Lectures will always be needed to provide the fundamentals of a given discipline, but students find lectures to be boring. When the environment is creative, they tend to learn more and this can be managed through discovery. A proposed 12 week research experience is much a medium. The research experience begins with typical lectures and slowly allows the learning to occur more and more within the student teams. Much more learning may 
occur between students than between a professor and a student. The professor provides an environment that allows learning to occur and the more creative that environment, the more learning. But this always needs to be tempered by a mentor or frustration sets in.

\section{References}

1. Alciatore, David and Histand, Michael (2003). Introduction to Mechatronics. McGraw Hill, New York, NY.

2. Mayer, Robert (2002). "A Source (of Energy) for Garcia"; Proceedings of 2002 American Society of Engineering Education Annual Conference and Exposition, Session 2133.

3. Takahasni, Patrick (2003). "Energy from the Sea: The Potential and Realities of Ocean Thermal Energy Conversion (OTEC)." Anton Bruun Memorial Lecture presented June 30, 2003 UNESCO House, Paris, France.

4. Vega, Louis A. (1992). "Economics of Ocean Thermal Energy Conversion (OTEC)" in R.J. Seymour, ed., Ocean Energy Recovery: The State of the Art, American Society of Civil Engineers, New York, NY.

\section{Acknowledgement}

I would like to thank Dr. George Watt who, with much patience, shows me what it means to be a professor of engineering. I would also like to thank my wife for enduring my endless conversations on mechatronics. 